\title{
Lobar analysis of collapsibility indices to assess functional lung volumes in COPD patients
}

This article was published in the following Dove Press journal:

International Journal of COPD

9 December 2014

Number of times this article has been viewed

\author{
Mariko Kitano' \\ Shingo Iwano' \\ Naozumi Hashimoto ${ }^{2}$ \\ Keiji Matsuo ${ }^{3}$ \\ Yoshinori Hasegawa ${ }^{2}$ \\ Shinji Naganawa' \\ 'Department of Radiology, \\ 2Department of Respiratory Medicine, \\ Graduate School of Medicine, \\ Nagoya University, Nagoya, Aichi, \\ Japan; ${ }^{3}$ Department of Radiology, \\ Ichinomiya Municipal Hospital, \\ Ichinomiya, Aichi, Japan
}

Correspondence: Shingo Iwano Department of Radiology, Nagoya University Graduate School of Medicine, 65 Tsurumai-cho, Shouwa-ku,

Nagoya 466-8550, Japan

Tel +8I 527442327

Fax +8I 527442335

Email iwano45@med.nagoya-u.ac.jp
Background: We investigated correlations between lung volume collapsibility indices and pulmonary function test (PFT) results and assessed lobar differences in chronic obstructive pulmonary disease (COPD) patients, using paired inspiratory and expiratory three dimensional (3D) computed tomography (CT) images.

Methods: We retrospectively assessed 28 COPD patients who underwent paired inspiratory and expiratory CT and PFT exams on the same day. A computer-aided diagnostic system calculated total lobar volume and emphysematous lobar volume (ELV). Normal lobar volume (NLV) was determined by subtracting ELV from total lobar volume, both for inspiratory phase $\left(\mathrm{NLV}_{\mathrm{I}}\right)$ and for expiratory phase $\left(\mathrm{NLV}_{\mathrm{E}}\right)$. We also determined lobar collapsibility indices: NLV collapsibility ratio $\left(\mathrm{NLV}_{\mathrm{CR}}\right)(\%)=\left(1-\mathrm{NLV}_{\mathrm{E}} / \mathrm{NLV}_{\mathrm{I}}\right) \times 100 \%$. Associations between lobar volumes and PFT results, and collapsibility indices and PFT results were determined by Pearson correlation analysis.

Results: $\mathrm{NLV}_{\mathrm{CR}}$ values were significantly correlated with PFT results. Forced expiratory volume in 1 second, measured as percent of predicted results $\left(\mathrm{FEV}_{1} \% \mathrm{P}\right)$ was significantly correlated with $\mathrm{NLV}_{\mathrm{CR}}$ values for the lower lobes $(P<0.01)$, whereas this correlation was not significant for the upper lobes $(P=0.05)$. $\mathrm{FEV}_{1} \% \mathrm{P}$ results were also moderately correlated with inspiratory, expiratory ELV $\left(\mathrm{ELV}_{\mathrm{I}, \mathrm{E}}\right)$ for the lower lobes $(P<0.05)$. In contrast, the ratio of the diffusion capacity for carbon monoxide to alveolar gas volume, measured as percent of predicted $\left(\mathrm{DL}_{\mathrm{CO}} /\right.$ $\left.\mathrm{V}_{\mathrm{A}} \% \mathrm{P}\right)$ results were strongly correlated with $\mathrm{ELV}_{\mathrm{I}}$ for the upper lobes $(P<0.001)$, whereas this correlation with $\mathrm{NLV}_{\mathrm{CR}}$ values was weaker for upper lobes $(P<0.01)$ and was not significant for the lower lobes $(P=0.26)$.

Conclusion: $\mathrm{FEV}_{1} \% \mathrm{P}$ results were correlated with NLV collapsibility indices for lower lobes, whereas $\mathrm{DL}_{\mathrm{CO}} / \mathrm{V}_{\mathrm{A}} \% \mathrm{P}$ results were correlated with NLV collapsibility indices and ELV for upper lobes. Thus, evaluating lobar NLV collapsibility might be useful for estimating pulmonary function in COPD patients.

Keywords: segmentation, emphysema, computed tomography, pulmonary function

\section{Background}

Chronic obstructive pulmonary disease (COPD) is characterized by persistent airflow limitation, which prevents lungs from collapsing appropriately for efficient oxygen exchange. This chronic airflow limitation is caused by a mixture of small airway disease (obstructive bronchiolitis) and parenchymal destruction (emphysema). ${ }^{1}$ Obstructive bronchiolitis causes air trapping, which, physiologically, is respiratory dead space even when normal pulmonary structure is preserved. Emphysematous areas and normal structures that include air-trapping areas are heterogeneously distributed and cause the symptoms associated with COPD. Precise functional 
assessments of each lobe are essential to determine COPD pathological mechanisms and subtypes, and might also be useful for lobectomy planning for lung cancer, especially for COPD patients.

To date, the severity and distributions of emphysematous areas have been assessed visually on high-resolution computed tomography (CT) images..$^{2-6}$ However, the recent development of three dimensional (3D) CT and computer aided diagnosis (CAD) has enabled $3 \mathrm{D}$ volumetry in vivo for each pulmonary lobe. ${ }^{7-12} \mathrm{~A}$ few studies that used 3D-CT volumetry of anatomic lobes showed that the distributions of emphysematous areas and pulmonary function test (PFT) results were different between the upper lobes and lower lobes. ${ }^{13-16}$ In addition, Kundu et al showed that total lobar volume (TLV) collapsibility indices, which were defined as the simple difference between CT computed inspiration (I) and expiration $(\mathrm{E})$ volumes, $(\mathrm{I}-\mathrm{E})$, and the relative measure of volume changes, $(\mathrm{I}-\mathrm{E}) / \mathrm{I}$, of lower lobes tended to be highly correlated with PFT results, using paired inspiratory and expiratory $\mathrm{CT} .{ }^{17}$ Respiration is gas exchange performed through ventilation of normal lung architecture. We hypothesized that the volume collapsibility of normal lobar volume (NLV) would show better correlations with PFT results than would the volume collapsibility of TLV and that the difference in NLV collapsibility might affect the difference in pulmonary function between upper and lower lobes. NLV can be measured in little time by eliminating emphysematous area from total lobe. ${ }^{13}$ The novelty of our study is that we evaluated NLV collapsibility, not TLV collapsibility, which Kundu et al used. ${ }^{17}$

Thus, in the study, we investigated correlations between NLV collapsibility indices of volume for each anatomic lobe and PFT results in COPD patients, and examined for differences in function between the upper and lower lobes.

\section{Methods}

\section{Subjects}

Our study population included 28 patients (26 males and two females; mean age, 72 years; age range, 57 to 86 years). We retrospectively investigated patients who were clinically diagnosed with COPD and whose raw data of paired inspiratory and expiratory CT examinations was available from September 2010 to September 2012. COPD was defined as a ratio of forced expiratory volume in 1 second $\left(\mathrm{FEV}_{1}\right)$ to forced vital capacity (FVC) $\left(\mathrm{FEV}_{1} / \mathrm{FVC}\right)$ of $<70 \%$ after inhaling a bronchodilator (beta-2 agonist). Patients were excluded if they had a history of lung surgery before their CT examination. Subjects were classified based on the five COPD severity categories established by Global Initiative for Chronic Obstructive Lung Disease (GOLD). ${ }^{1}$ These patients' characteristics are summarized in Table 1.

Our institutional review board approved this retrospective study of this cohort, for whom inspiratory and expiratory CT images were acquired (approval number 2013-0014). All subjects had previously given their informed consent for using their $\mathrm{CT}$ data for future research.

\section{CT examinations}

All CT images were acquired using a 64-row multidetector CT scanner (Aquilion ${ }^{\mathrm{TM}}$ 64; Toshiba Medical Systems Corp, Otawara, Tochigi, Japan). Each subject was scanned from the lung apex to the diaphragm during a breath-hold at end fullinspiration and at end normal-expiration. No contrast medium was administered. We used the following parameters: X-ray tube voltage of $120 \mathrm{kVp}$; automatic tube current at a maximum of $225 \mathrm{mAs}$; gantry rotation speed of 0.5 seconds; and beam collimation of $64 \times 0.5 \mathrm{~mm}$. Thin-section CT images were reconstructed at $0.5 \mathrm{~mm}$ thick slices with $0.5 \mathrm{~mm}$ intervals, using a standard spatial frequency reconstruction algorithm (FC11). Iterative reconstruction algorithms were not available.

\section{Measurements of whole lung and lobar volumes}

The digital imaging and communications in medicine (DICOM) data from 3D-CT were transferred to a workstation (Synapse $^{\text {TM }}$ Vincent version 3.1; Fujifilm Medical Systems, Tokyo, Japan). This workstation incorporated a lobar CAD system that was demonstrated to precisely measure lobar volumes in a previous study. ${ }^{18}$ This system automatically extracted right and left lungs, recognized lobar bronchi, and determined the locations of fissures (Figure 1). A radiologist with 4 years of experience interpreting chest CT images

Table I Subject characteristics

\begin{tabular}{lll}
\hline Parameter & Mean \pm SD & Range \\
\hline Age, years & $72 \pm 7$ & $57-86$ \\
BMI & $22.2 \pm 3.1$ & $16.8-28.9$ \\
Smoking index (pack-years) & $59.3 \pm 34.5$ & $3.8-171.0$ \\
TLC\%P (\%) & $101.62 \pm 12.08$ & $75.50-123.50$ \\
RV\%P (\%) & $107.62 \pm 24.56$ & $69.00-163.10$ \\
FEV,/FVC (\%) & $52.89 \pm 10.45$ & $33.64-67.45$ \\
FEV \%P (\%) & $79.74 \pm 22.56$ & $43.80-118.10$ \\
DL $_{\text {CO }} / \mathrm{N}_{\mathrm{A}} \% \mathrm{P}(\%)$ & $77.81 \pm 28.50$ & $32.10-132.80$ \\
\hline
\end{tabular}

Abbreviations: $\mathrm{BMI}$, body mass index; $\mathrm{DL}_{\mathrm{co}} / \mathrm{N}_{\mathrm{A}} \% \mathrm{P}$, ratio of the diffusion capacity for carbon monoxide to alveolar gas volume, measured as percent predicted; $\mathrm{FEV}_{1}$, forced expiratory volume in I second; $\mathrm{FEV}_{1} \% \mathrm{P}, \mathrm{FEV}$, measured as percent predicted; FVC, functional vital capacity; RV\%P, residual volume, measured as percent predicted; TLC\%P, total lung capacity, measured as percent predicted; SD, standard deviation. 


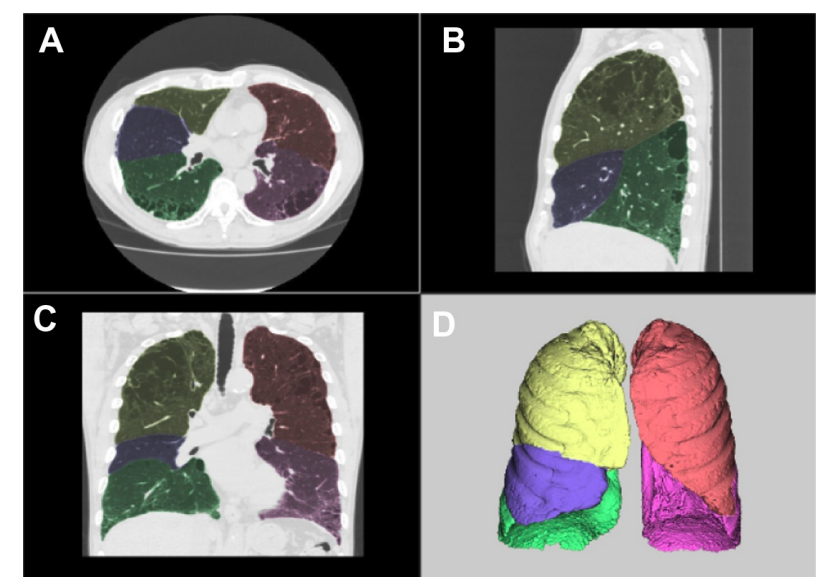

Figure I Examples of segmentation using automated CAD.

Notes: Axial (A), sagittal (B), and coronal (C) multiplanar reconstruction views and volume rendering.(D). Yellow is right upper lobe, blue is right middle lobe, green is right lower lobe, orange is left upper lobe, and pink is left lower lobe. Abbreviation: $\mathrm{CAD}$, computer-aided diagnosis.

(MK) verified the results of segmentation by CAD and made manual corrections by delineating fissures when the CAD system failed to properly identify fissures.

The extent of emphysema was estimated using a threshold technique, by quantifying the volume of voxels with an apparent X-ray attenuation value of $<-950 \mathrm{HU}$. We used the same threshold for an inspiratory scan and for an expiratory scan. Each lobar volume was defined as the TLV. The volume with low attenuation values, of $<-950$ $\mathrm{HU}$, in each lobe was defined as the emphysematous lobar volume (ELV). The workstation automatically quantified TLV and ELV for each lobe. NLV was determined by subtracting ELV from TLV.

We computed four lobar collapsibility indices:

$$
\Delta \mathrm{TLV}(\text { in liters })=\mathrm{TLV}_{\mathrm{I}}-\mathrm{TLV}_{\mathrm{E}}
$$

where $\mathrm{TLV}_{\mathrm{I}}$ is inspiratory $\mathrm{TLV}$ and $\mathrm{TLV}_{\mathrm{E}}$ is expiratory TLV;

$$
\operatorname{TLV}_{\mathrm{CR}}(\%)=\left(1-\mathrm{TLV}_{\mathrm{E}} / \mathrm{TLV}_{\mathrm{I}}\right) \times 100 \%
$$

where $\mathrm{TLV}_{\mathrm{CR}}$ is the TLV collapsibility ratio;

$$
\Delta \mathrm{NLV} \text { (in liters) }=\mathrm{NLV}_{\mathrm{I}}-\mathrm{NLV}_{\mathrm{E}}
$$

where $\mathrm{NLV}_{\mathrm{I}}$ is inspiratory NLV and $\mathrm{NLV}_{\mathrm{E}}$ is expiratory $\mathrm{NLV}$; and

$$
\mathrm{NLV}_{\mathrm{CR}}(\%)=\left(1-\mathrm{NLV}_{\mathrm{E}} / \mathrm{NLV}_{\mathrm{I}}\right) \times 100 \%
$$

where $\mathrm{NLV}_{\mathrm{CR}}$ is the NLV collapsibility ratio.

\section{PFTs}

Pulmonary function was determined on the same day as CT images were acquired, using a flow-sensing spirometer (FUDAC-77; Fukuda Denshi Co, Ltd, Tokyo, Japan). PFT results included total lung capacity, residual volume, $\mathrm{FEV}_{1} /$ $\mathrm{FVC}, \mathrm{FEV}_{1}$ measured as percent predicted $\left(\mathrm{FEV}_{1} \% \mathrm{P}\right)$, and the ratio of the single-breath diffusion capacity for carbon monoxide to alveolar gas volume, measured as percent predicted $\left(\mathrm{DL} \mathrm{CO}_{\mathrm{A}} / \mathrm{V}_{\mathrm{A}} \% \mathrm{P}\right)$. These results are summarized in Table 1.

\section{Statistical analysis}

Results were given as mean \pm standard deviation for TLV, ELV, and NLV, for the inspiratory and expiratory phases. These results were compared for whole lung, each lobe, upper lobes, and lower lobes. Upper lobes included the right upper lobe (RUL), right middle lobe (RML), and left upper lobe (LUL). Lower lobes included the right lower lobe (RLL) and left lower lobe (LLL). Subsequently, we determined $\triangle T L V$, $\mathrm{TLV}_{\mathrm{CR}}, \Delta \mathrm{NLV}$, and $\mathrm{NLV}_{\mathrm{CR}}$ for whole lung, each lobe, upper lobes, and lower lobes. Pearson correlation coefficients $(r)$ were determined to assess the associations between the PFT results and CT-derived indices.

Statistical analyses were done using Excel ${ }^{\circledR} 2010$ (Microsoft Corp, Redmond, WA, USA). A $P$-value of $<0.05$ was considered significant.

\section{Results}

Table 1 summarizes our study subjects' characteristics. Based on GOLD staging categories, these 28 patients were classified as: stage $1(n=16)$; stage $2(n=10)$; and stage $3(n=2)$. One subject had interstitial pneumonia. Regarding smoking status, three subjects were current smokers, and 25 were former smokers.

Table 2 shows the mean values of lobar volumes and collapsibility indices from 3D-CT volumetry. The ELV percentages of upper lobes were slightly larger than those of lower lobes. $\triangle \mathrm{TLV}$ and $\mathrm{TLV}_{\mathrm{CR}}$ of lower lobes were larger than those of upper lobes. Similarly, $\triangle \mathrm{NLV}$ and $\mathrm{NLV}_{\mathrm{CR}}$ of lower lobes were larger than those of upper lobes.

\section{Whole-lung analysis}

$\mathrm{TLV}_{\mathrm{I}}$ for whole lung was strongly correlated with total lung capacity $(r=0.90, P<0.0001)$ and $\mathrm{TLV}_{\mathrm{E}}$ for whole lung with residual volume $(r=0.73, P<0.0001)$.

As shown in Table 3, $\triangle \mathrm{NLV}$ for whole lung and $\mathrm{NLV}_{\mathrm{CR}}$ for whole lung were positively correlated with PFT results ( $r=0.41$ to 0.52 , all $P<0.05$ ), and ELV for whole lung - both for inspiratory phase and expiratory phase $\left(\mathrm{ELV}_{\mathrm{I}, \mathrm{E}}\right)$ - were negatively correlated with PFT results $(r=-0.62$ to -0.38 , 
Table 2 Lobar volumes and collapsibility indices derived from 3D-CT volumetry

\begin{tabular}{|c|c|c|c|c|c|c|c|c|}
\hline & Whole lung & RUL & RML & RLL & LUL & LLL & Upper lobes & Lower lobes" \\
\hline $\operatorname{TLV}_{1}(\mathrm{~L})$ & $5.32 \pm 0.88$ & $1.13 \pm 0.26$ & $0.43 \pm 0.18$ & $1.28 \pm 0.29$ & $1.33 \pm 0.23$ & $1.15 \pm 0.36$ & $2.89 \pm 0.53$ & $2.43 \pm 0.58$ \\
\hline $\operatorname{TLV}_{\mathrm{E}}(\mathrm{L})$ & $3.54 \pm 0.84$ & $0.79 \pm 0.24$ & $0.33 \pm 0.14$ & $0.77 \pm 0.27$ & $0.94 \pm 0.24$ & $0.7 I \pm 0.25$ & $2.06 \pm 0.52$ & $1.48 \pm 0.46$ \\
\hline $\operatorname{ELV}_{1}(\mathrm{~L})$ & $1.06 \pm 0.89$ & $0.23 \pm 0.21$ & $0.09 \pm 0.08$ & $0.21 \pm 0.20$ & $0.29 \pm 0.21$ & $0.24 \pm 0.32$ & $0.61 \pm 0.45$ & $0.45 \pm 0.50$ \\
\hline $\mathrm{ELV}_{\mathrm{E}}(\mathrm{L})$ & $0.28 \pm 0.36$ & $0.08 \pm 0.12$ & $0.03 \pm 0.03$ & $0.04 \pm 0.05$ & $0.09 \pm 0.12$ & $0.05 \pm 0.12$ & $0.19 \pm 0.25$ & $0.09 \pm 0.17$ \\
\hline $\operatorname{ELV}_{1}(\%)$ & $18.8 \pm 13.6$ & $18.6 \pm 14.6$ & $21.0 \pm 15.4$ & $15.5 \pm 12.6$ & $21.4 \pm 14.8$ & $17.8 \pm 16.1$ & $20.4 \pm 14.0$ & $16.8 \pm 14.2$ \\
\hline $\operatorname{ELV}_{E}(\%)$ & $6.9 \pm 8.4$ & $7.9 \pm 10.2$ & $8.2 \pm 10.2$ & $3.9 \pm 5.8$ & $8.0 \pm 9.2$ & $5.6 \pm 10.6$ & $8.0 \pm 9.0$ & $4.9 \pm 8.4$ \\
\hline $\mathrm{NLV}_{1}(\mathrm{~L})$ & $4.25 \pm 0.77$ & $0.90 \pm 0.22$ & $0.34 \pm 0.14$ & $1.07 \pm 0.26$ & $1.03 \pm 0.21$ & $0.91 \pm 0.23$ & $2.28 \pm 0.47$ & $1.98 \pm 0.45$ \\
\hline $\mathrm{NLV}_{\mathrm{E}}(\mathrm{L})$ & $3.26 \pm 0.69$ & $0.72 \pm 0.18$ & $0.31 \pm 0.13$ & $0.74 \pm 0.25$ & $0.85 \pm 0.19$ & $0.65 \pm 0.20$ & $\mathrm{I} .87 \pm 0.40$ & $1.39 \pm 0.4 \mathrm{I}$ \\
\hline$\Delta \mathrm{TLV}^{*}(\mathrm{~L})$ & I. $.78 \pm 0.78$ & $0.34 \pm 0.15$ & $0.10 \pm 0.07$ & $0.5 I \pm 0.23$ & $0.39 \pm 0.18$ & $0.44 \pm 0.24$ & $0.83 \pm 0.37$ & $0.95 \pm 0.46$ \\
\hline $\operatorname{TLV}_{\mathrm{CR}} * *(\%)$ & $33.3 \pm 12.5$ & $29.4 \pm 11.8$ & $22.8 \pm 11.5$ & $39.8 \pm 14.8$ & $29.5 \pm 13.1$ & $38.1 \pm 14.0$ & $28.7 \pm 11.9$ & $38.9 \pm 14.1$ \\
\hline$\Delta \mathrm{NLV}^{\dagger}(\mathrm{L})$ & $0.99 \pm 0.6 \mathrm{I}$ & $0.19 \pm 0.13$ & $0.03 \pm 0.04$ & $0.33 \pm 0.20$ & $0.18 \pm 0.14$ & $0.26 \pm 0.19$ & $0.40 \pm 0.29$ & $0.59 \pm 0.38$ \\
\hline $\operatorname{NLV}_{C R}^{\ddagger}(\%)$ & $22.9 \pm 12.4$ & $19.9 \pm 12.5$ & $9.1 \pm 12.7$ & $30.9 \pm 16.0$ & $16.7 \pm 12.5$ & $27.4 \pm 17.2$ & $16.9 \pm 1 \mid .4$ & $29.2 \pm 16.1$ \\
\hline
\end{tabular}

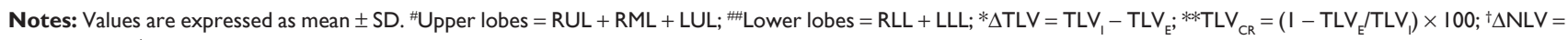
$N L V_{1}-N L V_{E} ;{ }^{\ddagger} N L V_{C R}=\left(I-N L V_{E} / N L V_{1}\right) \times 100$

Abbreviations: 3D-CT, three-dimensional computed tomography; CR, collapsibility ratio; E, expiratory; ELV, emphysematous lobar volume; I, inspiratory; LLL, left lower lobe; LUL, left upper lobe; NLV, normal lobar volume; RLL, right lower lobe; RML, right middle lobe; RUL, right upper lobe; TLV, total lobar volume.

all $P<0.05)$. In contrast, $\mathrm{TLV}_{\mathrm{I}}, \mathrm{NLV}_{\mathrm{I}, \mathrm{E}}, \Delta \mathrm{TLV}$, and $\mathrm{TLV}_{\mathrm{CR}}$ for whole lung were not correlated with PFT results. TLV $_{\mathrm{E}}$ for whole lung was correlated with $\mathrm{FEV}_{1} \% \mathrm{P}$ but was not correlated with $\mathrm{DL}_{\mathrm{CO}} / \mathrm{V}_{\mathrm{A}} \% \mathrm{P}$.

\section{Lung lobar analysis}

Tables 4 and 5 summarize the correlations found between PFT results and CT-derived indices.

$\mathrm{FEV}_{1} \% \mathrm{P}$ results were significantly correlated with $\mathrm{NLV}_{\mathrm{CR}}$ values for RLL $(r=0.56, P<0.01)$ and $\operatorname{LLL}(r=0.57, P<0.01)$. $\mathrm{FEV}_{1} \% \mathrm{P}$ results were also significantly correlated with $\triangle \mathrm{NLV}$ and $\mathrm{ELV}_{\mathrm{I}, \mathrm{E}}$ for RLL ( $r=-0.41$ to 0.47 , all $P<0.05$ ) and LLL ( $r=-0.45$ to 0.50 , all $P<0.05)$.

Table 3 Pearson correlation coefficients between pulmonary function and CT-derived indices for whole lung

\begin{tabular}{|c|c|c|c|c|}
\hline & \multicolumn{2}{|c|}{ FEV $\%$ P } & \multicolumn{2}{|c|}{$\mathbf{D L} \mathrm{L}_{\mathrm{CO}} / \mathbf{V}_{\mathrm{A}} \% \mathbf{P}$} \\
\hline & $r$ & $P$-value & $r$ & $P$-value \\
\hline $\operatorname{TLV}_{1}(\mathrm{~L})$ & -0.31 & 0.112 & -0.32 & 0.093 \\
\hline $\operatorname{TLV}_{E}(L)$ & $-0.4 \mathrm{I}$ & 0.032 & -0.30 & 0.122 \\
\hline $\operatorname{ELV}_{1}(\%)$ & -0.38 & 0.049 & -0.60 & 0.001 \\
\hline $\operatorname{ELV}_{\mathrm{E}}(\%)$ & -0.43 & 0.022 & -0.62 & 0.000 \\
\hline $\mathrm{NLV}_{1}(\mathrm{~L})$ & 0.12 & 0.554 & 0.26 & 0.175 \\
\hline $\mathrm{NLV}_{\mathrm{E}}(\mathrm{L})$ & -0.28 & 0.144 & -0.07 & 0.734 \\
\hline$\Delta \operatorname{TLV}^{*}(\mathrm{~L})$ & 0.09 & 0.645 & -0.04 & 0.826 \\
\hline $\operatorname{TLV}_{C R} * *(\%)$ & 0.26 & 0.185 & 0.11 & 0.579 \\
\hline$\Delta \mathrm{NLV}^{\dagger}(\mathrm{L})$ & 0.47 & 0.012 & $0.4 I$ & 0.031 \\
\hline $\operatorname{NLV}_{C R}^{\ddagger}(\%)$ & 0.52 & 0.004 & 0.41 & 0.028 \\
\hline
\end{tabular}

Notes: ${ }^{*} \Delta \mathrm{TLV}=\mathrm{TLV}_{1}-\mathrm{TLV}_{\mathrm{E}} ; * * \mathrm{TLV}_{\mathrm{CR}}=\left(1-\mathrm{TLV}_{\mathrm{E}} / \mathrm{TLV}_{1}\right) \times 100 ;{ }^{\dagger} \Delta \mathrm{NLV}=\mathrm{NLV}_{1}-$ $\mathrm{NLV}_{\mathrm{E}} ; \mathrm{NLV}_{\mathrm{CR}}=\left(1-\mathrm{NLV}_{\mathrm{E}} / \mathrm{NLV}\right) \times 100$.

Abbreviations: $\mathrm{CR}$, collapsibility ratio; $\mathrm{CT}$, computed tomography; $\mathrm{DL}_{\mathrm{CO}} / \mathrm{N}_{\mathrm{A}} \% \mathrm{P}$, ratio of the diffusion capacity for carbon monoxide to alveolar gas volume, measured as percent predicted; E, expiratory; ELV, emphysematous lobar volume; $F E V$ \%P, forced expiratory volume in I second, measured as percent predicted; I, inspiratory; NLV, normal lobar volume; $r$, Pearson correlation coefficient; TLV, total lobar volume.
$\mathrm{DL}_{\mathrm{CO}} / \mathrm{V}_{\mathrm{A}} \% \mathrm{P}$ results were significantly correlated with $\triangle \mathrm{NLV}$ and $\mathrm{NLV}_{\mathrm{CR}}$ values for RUL ( $r=0.56$ to 0.59 , all $P<0.01)$ and LUL ( $r=0.50$ to 0.52 , all $P<0.01)$. $\mathrm{DL}_{\mathrm{CO}} / \mathrm{V}_{\mathrm{A}} \% \mathrm{P}$ results were strongly and negatively correlated with $\mathrm{ELV}_{\mathrm{I}}$ for RUL $(r=-0.68, P<0.001)$ and LUL $(r=-0.63, P<0.001)$.

\section{Upper lobes vs lower lobes}

We summed the RUL, RML, and LUL volumes for the upper lobes, and RLL and LLL volumes for the lower lobes (Table 6 and Figures 2-5), in order to clarify their anatomical differences.

$\mathrm{FEV}_{1} \% \mathrm{P}$ results were significantly and positively correlated with $\mathrm{NLV}_{\mathrm{CR}}$ values for the lower lobes $(r=0.58, P<0.01)$ (Figure $2 \mathrm{~B})$, whereas this correlation was not significant for the upper lobes $(r=0.37, P=0.05)$ (Figure $2 \mathrm{~A}$ ). $\mathrm{FEV}_{1} \% \mathrm{P}$ results were also positively correlated with $\triangle \mathrm{NLV}$ for the lower lobes $(r=0.49, P<0.01)$ and negatively with $\mathrm{ELV}_{\mathrm{I}, \mathrm{E}}$ for the lower lobes (all $r=-0.44$, all $P<0.05$ ) (Figure $3 \mathrm{~A}$ and $\mathrm{B}$ ).

In contrast, $\mathrm{DL}_{\mathrm{CO}} / \mathrm{V}_{\mathrm{A}} \% \mathrm{P}$ results were significantly and positively correlated with $\triangle \mathrm{NLV}$ and $\mathrm{NLV}_{\mathrm{CR}}$ values for the upper lobes ( $r=0.54$ and 0.58 , respectively, all $P<0.01$ ) (Figure 4A), whereas this correlation was not significant for the lower lobes $(r=0.24$ and 0.22 , respectively, $P=0.22$ and 0.26 , respectively) (Figure $4 \mathrm{~B}$ ). Furthermore, $\mathrm{DL}_{\mathrm{CO}} / \mathrm{V}_{\mathrm{A}} \% \mathrm{P}$ results and $\mathrm{ELV}_{\mathrm{I}, \mathrm{E}}$ showed strong negative correlations for the upper lobes ( $r=-0.67$ to -0.62 , all $P<0.001$ ) (Figure 5A) and moderate negative correlations for the lower lobes $(r=-0.49$ to -0.46 , all $P<0.05$ ) (Figure 5B).

\section{Discussion}

The results of the present study demonstrated that pulmonary function might be different between the upper and lower 
Table 4 Pearson correlation coefficients between $\mathrm{FEV}, \% \mathrm{P}$ results and $\mathrm{CT}$-derived indices for each lobe

\begin{tabular}{|c|c|c|c|c|c|c|c|c|c|c|}
\hline & \multicolumn{2}{|l|}{ RUL } & \multicolumn{2}{|l|}{ RML } & \multicolumn{2}{|l|}{ RLL } & \multicolumn{2}{|l|}{ LUL } & \multicolumn{2}{|l|}{ LLL } \\
\hline & $r$ & $P$-value & $r$ & $P$-value & $r$ & $P$-value & $r$ & $P$-value & $r$ & $P$-value \\
\hline $\operatorname{TLV}_{1}(\mathrm{~L})$ & -0.36 & 0.060 & 0.09 & 0.656 & -0.27 & 0.171 & -0.12 & 0.535 & -0.24 & 0.213 \\
\hline $\operatorname{TLV}_{\mathrm{E}}(\mathrm{L})$ & -0.34 & 0.079 & -0.02 & 0.935 & -0.42 & 0.024 & -0.19 & 0.328 & $-0.4 \mathrm{I}$ & 0.032 \\
\hline $\operatorname{ELV}_{1}(\%)$ & -0.34 & 0.074 & -0.27 & 0.171 & $-0.4 \mathrm{I}$ & 0.030 & -0.26 & 0.179 & -0.45 & 0.016 \\
\hline $\operatorname{ELV}_{E}(\%)$ & -0.36 & 0.062 & -0.52 & 0.004 & -0.40 & 0.034 & -0.28 & 0.150 & -0.44 & 0.020 \\
\hline $\mathrm{NLV}_{1}(\mathrm{~L})$ & -0.05 & 0.807 & 0.18 & 0.366 & 0.00 & 0.988 & 0.16 & 0.427 & 0.18 & 0.347 \\
\hline $\operatorname{NLV}_{E}(L)$ & -0.24 & 0.213 & 0.09 & 0.651 & -0.38 & 0.046 & -0.10 & 0.623 & -0.26 & 0.176 \\
\hline$\Delta \mathrm{TLV}^{*}(\mathrm{~L})$ & -0.09 & 0.654 & 0.25 & 0.197 & 0.16 & 0.429 & 0.09 & 0.632 & 0.05 & 0.796 \\
\hline $\operatorname{TLV}_{\mathrm{CR}}{ }^{* *}(\%)$ & 0.12 & 0.544 & 0.29 & 0.137 & 0.34 & 0.073 & 0.14 & 0.485 & 0.35 & 0.066 \\
\hline$\Delta \mathrm{NLV}^{\dagger}(\mathrm{L})$ & 0.25 & 0.202 & 0.34 & 0.080 & 0.47 & 0.013 & 0.36 & 0.064 & 0.50 & 0.007 \\
\hline $\operatorname{NLV}_{C R}{ }^{\ddagger}(\%)$ & 0.34 & 0.081 & 0.29 & 0.132 & 0.56 & 0.002 & 0.40 & 0.036 & 0.57 & 0.002 \\
\hline
\end{tabular}

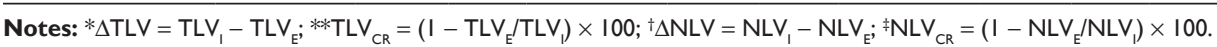

Abbreviations: $\mathrm{CR}$, collapsibility ratio; $\mathrm{CT}$, computed tomography; $\mathrm{E}$, expiratory; ELV, emphysematous lobar volume; FEV \%P, forced expiratory volume in I second, measured as percent predicted; I, inspiratory; LLL, left lower lobe; LUL, left upper lobe; NLV, normal lobar volume; $r$, Pearson correlation coefficient; RLL, right lower lobe; RML, right middle lobe; RUL, right upper lobe; TLV, total lobar volume.

lobes in COPD patients. $\mathrm{FEV}_{1} \% \mathrm{P}$ results were correlated significantly with lower lobe collapsibility indices, whereas $\mathrm{DL}_{\mathrm{CO}} / \mathrm{V}_{\mathrm{A}} \% \mathrm{P}$ results were correlated strongly with collapsibility indices and ELV values for upper lobes. These functional differences between individual lung lobes may be useful for early detection and for assessing the severity of COPD.

We showed that $\mathrm{FEV}_{1} \% \mathrm{P}$ results measured during forced expiration were more strongly correlated with lower lobe collapsibility indices than with those of upper lobes. This was consistent with the results in previous reports. Matsuo et al studied the correlation between PFT results and lobar NLV, using an inspiratory multiple detector CT scan, and reported that lower lobe volumes were more strongly correlated with $\mathrm{FEV}_{1}$ results than were those of upper lobes. ${ }^{13}$ Kundu et al examined the correlation between PFT results and the collapsibility indices of each lobar TLV, using inspiratory and expiratory multiple detector $\mathrm{CT}$ scan, and reported that lower lobe volume changes were more strongly correlated with $\mathrm{FEV}_{1} \% \mathrm{P}$ than were those of upper lobes. ${ }^{17}$ These results indicate that lower lobe collapsibility more directly affects $\mathrm{FEV}_{1}$. The reason is that the lower lobes can collapse more readily than can the upper lobes during a forced expiration because the intrathoracic pressure is less negative in the lower lung field than in the upper lung field. ${ }^{19}$ In general, emphysematous areas are found only in the upper lobes at earlier COPD disease stages and then, spread to lower lobes as the disease progresses. ${ }^{20}$ The upper lobe-predominant distribution of emphysematous areas and our results explain why $\mathrm{FEV}_{1}$ as measured by PFT may not detect the very early stage of COPD.

$\mathrm{FEV}_{1} \% \mathrm{P}$ results also showed negative correlations with lower lobe $\mathrm{ELV}_{\mathrm{I}, \mathrm{E}}$. We speculated the reason would be gravitational difference within lung. Airflow limitation is more

Table 5 Pearson correlation coefficients between $\mathrm{DL}_{\mathrm{CO}} / \mathrm{V}_{\mathrm{A}} \% \mathrm{P}$ results and CT-derived indices for each lobe

\begin{tabular}{|c|c|c|c|c|c|c|c|c|c|c|}
\hline & \multicolumn{2}{|l|}{ RUL } & \multicolumn{2}{|l|}{ RML } & \multicolumn{2}{|l|}{ RLL } & \multicolumn{2}{|l|}{ LUL } & \multicolumn{2}{|l|}{ LLL } \\
\hline & $r$ & $P$-value & $r$ & $P$-value & $r$ & $P$-value & $r$ & $P$-value & $r$ & $P$-value \\
\hline $\operatorname{TLV}_{1}(\mathrm{~L})$ & -0.29 & 0.138 & -0.12 & 0.539 & 0.00 & 1.000 & -0.28 & 0.152 & -0.35 & 0.070 \\
\hline $\operatorname{TLV}_{\mathrm{E}}(\mathrm{L})$ & -0.31 & 0.106 & -0.17 & 0.399 & -0.02 & 0.908 & -0.29 & 0.136 & -0.32 & 0.092 \\
\hline $\operatorname{ELV}_{1}(\%)$ & -0.68 & 0.000 & -0.44 & 0.019 & -0.44 & 0.019 & -0.63 & 0.000 & -0.47 & 0.012 \\
\hline $\operatorname{ELV}_{E}(\%)$ & -0.56 & 0.002 & -0.5 I & 0.005 & -0.52 & 0.004 & -0.59 & 0.001 & -0.45 & 0.016 \\
\hline $\mathrm{NLV}_{1}(\mathrm{~L})$ & 0.25 & 0.193 & 0.05 & 0.813 & 0.29 & 0.135 & 0.28 & 0.144 & 0.03 & 0.897 \\
\hline $\mathrm{NLV}_{\mathrm{E}}(\mathrm{L})$ & -0.10 & 0.620 & -0.05 & 0.803 & 0.08 & 0.674 & -0.07 & 0.724 & -0.15 & 0.453 \\
\hline$\Delta \mathrm{TLV}^{*}(\mathrm{~L})$ & 0.00 & 0.992 & 0.02 & 0.930 & 0.03 & 0.894 & 0.02 & 0.915 & -0.19 & 0.343 \\
\hline $\operatorname{TLV}_{\mathrm{CR}} * *(\%)$ & 0.19 & 0.321 & 0.07 & 0.740 & 0.05 & 0.794 & 0.09 & 0.639 & 0.03 & 0.893 \\
\hline$\Delta \mathrm{NLV}^{\dagger}(\mathrm{L})$ & 0.56 & 0.002 & 0.31 & 0.111 & 0.28 & 0.154 & 0.50 & 0.006 & 0.19 & 0.344 \\
\hline $\operatorname{NLV}_{C R}{ }^{\ddagger}(\%)$ & 0.59 & 0.001 & 0.31 & 0.111 & 0.21 & 0.281 & 0.52 & 0.004 & 0.22 & 0.256 \\
\hline
\end{tabular}

Notes: ${ }^{*} \Delta \mathrm{TLV}=\mathrm{TLV}_{1}-\mathrm{TLV}_{\mathrm{E}} ; * * \mathrm{TLV}_{\mathrm{CR}}=\left(1-\mathrm{TLV}_{\mathrm{E}} / \mathrm{TLV}_{1}\right) \times 100 ;{ }^{\dagger} \Delta \mathrm{NLV}=\mathrm{NLV}_{1}-\mathrm{NLV}_{\mathrm{E}} ;{ }^{\mathrm{N} L V_{\mathrm{CR}}}=\left(1-\mathrm{NLV}_{\mathrm{E}} / \mathrm{NLV}_{1}\right) \times 100$

Abbreviations: $\mathrm{CR}$, collapsibility ratio; $\mathrm{CT}$, computed tomography; $\mathrm{DL}_{\mathrm{CO}} / \mathrm{V}_{\mathrm{A}} \% \mathrm{P}$, ratio of the diffusion capacity for carbon monoxide to alveolar gas volume, measured as percent predicted; E, expiratory; ELV, emphysematous lobar volume; I, inspiratory; LLL, left lower lobe; LUL, left upper lobe; NLV, normal lobar volume; $r$, Pearson correlation coefficient; RLL, right lower lobe; RML, right middle lobe; RUL, right upper lobe; TLV, total lobar volume. 
Table 6 Pearson correlation coefficients between pulmonary function and CT-derived indices for upper lobes and for lower lobes

\begin{tabular}{|c|c|c|c|c|c|c|c|c|}
\hline & \multicolumn{4}{|c|}{$\mathrm{FEV}_{1} \% \mathrm{P}$} & \multicolumn{4}{|c|}{$\mathrm{DL}_{\mathrm{Co}} / \mathbf{V}_{\mathrm{A}} \% \mathbf{P}$} \\
\hline & \multicolumn{2}{|c|}{$\begin{array}{l}\text { Upper } \\
\text { lobes }\end{array}$} & \multicolumn{2}{|c|}{$\begin{array}{l}\text { Lower } \\
\text { lobes }\end{array}$} & \multicolumn{2}{|c|}{$\begin{array}{l}\text { Upper } \\
\text { lobes }\end{array}$} & \multicolumn{2}{|c|}{ Lower lobes } \\
\hline & $r$ & $P$-value & $r$ & $P$-value & $r$ & $P$-value & $r$ & $P$-value \\
\hline $\mathrm{ELV}_{1}(\%)$ & -0.30 & 0.123 & -0.44 & 0.018 & -0.67 & 0.000 & -0.46 & 0.013 \\
\hline $\operatorname{ELV}_{\mathrm{E}}(\%)$ & -0.36 & 0.059 & -0.44 & +0.020 & -0.62 & 0.000 & -0.49 & 0.009 \\
\hline $\begin{array}{l}\Delta \mathrm{NLV}^{\dagger} \\
(\mathrm{L})\end{array}$ & 0.33 & 0.083 & 0.49 & 0.008 & 0.54 & 0.003 & 0.24 & 0.224 \\
\hline $\begin{array}{l}\mathrm{NLV}_{C R}{ }^{\ddagger} \\
(\%)\end{array}$ & 0.37 & 0.050 & 0.58 & 0.001 & 0.58 & 0.001 & 0.22 & 0.255 \\
\hline
\end{tabular}

Notes: \#Upper lobes = RUL + RML + LUL; "\#ower lobes = RLL + LLL; ${ }^{\dagger} \Delta N L V=$ $N L V_{1}-N_{E} ; V_{E} ; \operatorname{NLV}_{C R}=\left(I-N L V_{E} / N L V_{1}\right) \times 100$.

Abbreviations: $C R$, collapsibility ratio; $C T$, computed tomography; $\mathrm{DL}_{\mathrm{CO}} / \mathrm{N}_{\mathrm{A}} \% \mathrm{P}$, ratio of the diffusion capacity for carbon monoxide to alveolar gas volume, measured as percent predicted; E, expiratory; ELV, emphysematous lobar volume; I, inspiratory; LLL, left lower lobe; LUL, left upper lobe; NLV, normal lobar volume; $r$, Pearson correlation coefficient; RLL, right lower lobe; RML, right middle lobe; RUL, right upper lobe; $\mathrm{FEV} \% \mathrm{P}$, forced expiratory volume in I second, measured as percent predicted.

influenced by the lower lobes because airway closure begins in the lower lobes while in a sitting or standing position, due to gravitational differences in the lung. ${ }^{21}$ Our result regarding ELV was the same as that of Saitoh et al, who studied the correlations between percentage of extent of emphysema in each lobe and PFT results in 50 emphysema patients, using helical CT. ${ }^{22}$

Correlations between the $\mathrm{DL}_{\mathrm{CO}} / \mathrm{V}_{\mathrm{A}} \% \mathrm{P}$ results and collapsibility indices were different between the upper and lower lobes in COPD patients. The $\mathrm{DL}_{\mathrm{CO}} / \mathrm{V}_{\mathrm{A}} \% \mathrm{P}$ results showed stronger positive correlations with upper lobe collapsibility indices than with those of lower lobes. Similarly, $\mathrm{DL}_{\mathrm{CO}} / \mathrm{V}_{\mathrm{A}} \% \mathrm{P}$ results showed strong negative correlations with upper lobe
$\mathrm{ELV}_{\mathrm{I}, \mathrm{E}}$. Emphysema progression decreases both pulmonary collapsibility and the surface area for gas exchange, and the decrease of the surface area reduces $\mathrm{DL}_{\mathrm{CO}} / \mathrm{V}_{\mathrm{A}} \% \mathrm{P}$ values. Accordingly, ELV might be a confounding factor in the correlation of collapsibility indices and $\mathrm{DL}_{\mathrm{CO}} / \mathrm{V}_{\mathrm{A}} \% \mathrm{P}$ values. We speculated the main reason for our result would be the upper lobe-predominant emphysema distribution, and the second reason could be the anatomic differences between each lobe.

Parr et al also reported that predominantly apical emphysema was associated with greater impairment of gas exchange than predominantly basal emphysema. ${ }^{23}$ They suggested the main reason was that subjects with apical emphysema would be unlikely to maintain gas exchange by recruitment of inferior normal lung units because these would already be well perfused, while subjects with basal emphysema could maintain $\mathrm{DL}_{\mathrm{CO}} / \mathrm{V}_{\mathrm{A}}$ through the recruitment of underperfused disease-free lung units in the upper regions. This recruitment would result from hypoxic pulmonary vasoconstriction - the theory to explain the blood flow redistribution in response to hypoxia. ${ }^{24}$ In a sitting position, when spirometry is performed, the upper lobes show a low blood flow as compared with the lower lobes, due to gravitational differences within the lung, ${ }^{19}$ but this gradient of perfusion could be altered by hypoxic pulmonary vasoconstriction. We presumed blood flow redistribution was effective when lower lobes were hypoxic but wasn't effective enough when upper lobes were hypoxic since lower lobes would already be well perfused. Thus, the gas exchange capability of the upper lobes can be easily impaired if the surface area of the upper lobes decreases because perfusion cannot compensate for this.
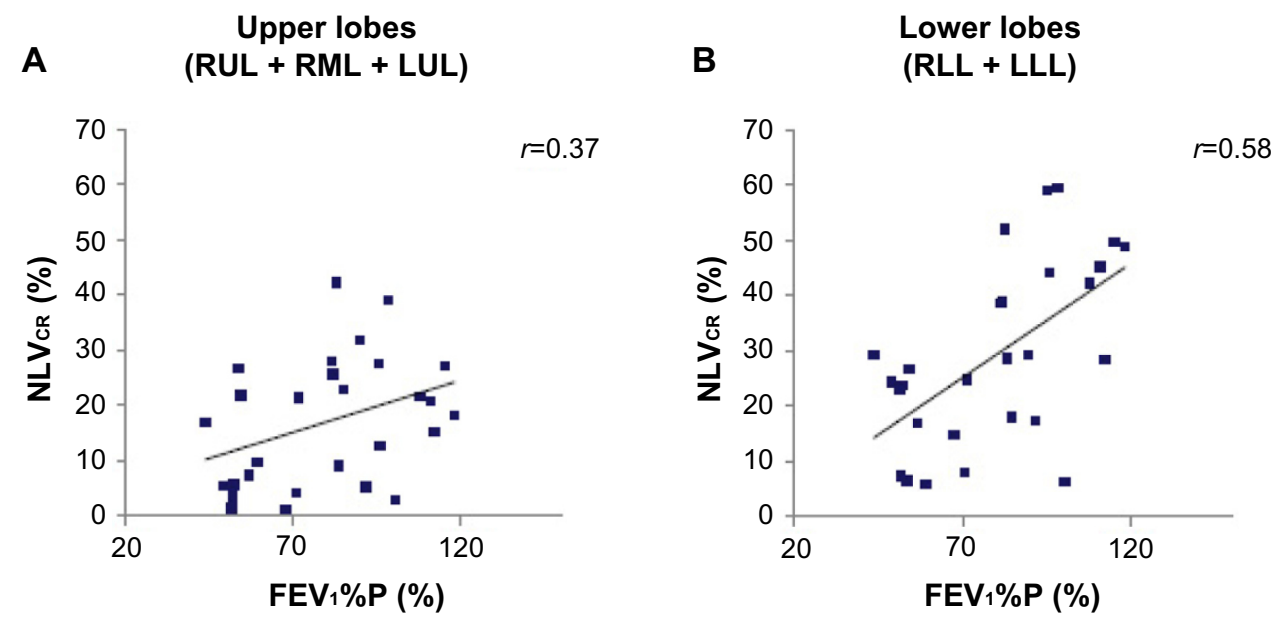

Figure 2 Correlations between $\mathrm{NLV}_{\mathrm{CR}}$ of upper lobes $(\mathbf{A})$, lower lobes $(\mathbf{B})$, and $\mathrm{FEV}, \% \mathrm{P}$ results.

Notes: $F E V_{1} \% P$ results were significantly correlated with $\mathrm{NLV}_{C R}$ values for the lower lobes (B), whereas this correlation was not significant for the upper lobes (A).

Abbreviations: $\mathrm{FEV}_{1} \% \mathrm{P}$, forced expiratory volume in I second measured as percent predicted; LLL, left lower lobe; LUL, left upper lobe; NLV $\mathrm{CR}$, normal lobar volume collapsibility ratio; RML, right middle lobe; RLL, right lower lobe; RUL, right upper lobe. 


\section{A}

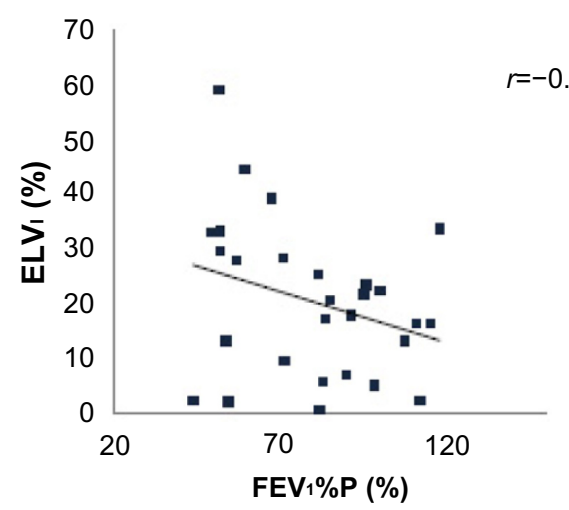

B $\quad \begin{aligned} & \text { Lower lobes } \\ & \text { (RLL + LLL) }\end{aligned}$

B $\quad \begin{aligned} & \text { Lower lobes } \\ & (\mathrm{RLL}+\mathrm{LLL})\end{aligned}$

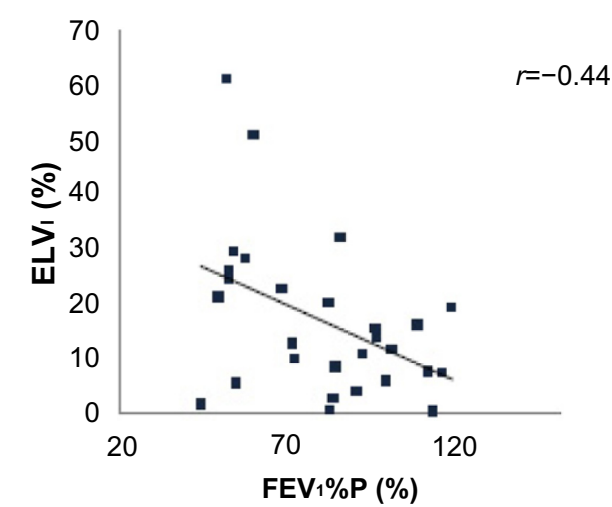

Figure 3 Correlations between ELV, of upper lobes $(\mathbf{A})$ and lower lobes $(\mathbf{B})$ and $\mathrm{FEV} \% \mathrm{P}$ results.

Notes: $\mathrm{FEV}, \% \mathrm{P}$ results were significantly correlated with $\mathrm{ELV}_{1}$ values for the lower lobes (B), whereas this correlation was not significant for the upper lobes (A).

Abbreviations: ELV, inspiratory emphysematous lobar volume; FEV \% , forced expiratory volume in I second measured as percent predicted; LLL, left lower lobe; LUL, left upper lobe; RLL, right lower lobe; RML, right middle lobe; RUL, right upper lobe.

In COPD patients, lung cancer is often a complication. ${ }^{25-27}$ The first treatment choice for early stage lung cancer is thoracic surgery. ${ }^{28}$ Postoperative pulmonary function after lobectomy, which is the most commonly performed surgery for lung cancer, depends upon the volume of the residual anatomical lobe. ${ }^{29-31}$ To date, this has been predicted based on the hypothesis that the function of each anatomic lobe is uniform. However, our results showed that the function of each lobe was so different that anatomic information should be added to volumetric information in order to more accurately predict postoperative pulmonary function in COPD patients. As it is now, many researchers reported actual postoperative pulmonary function results were better than predicted postoperative values based on numbers of resected subsegments/segments in COPD patients and in non-COPD subjects. ${ }^{32-36}$ These researchers suggested the reason would be the refractory improvement of remaining lung function by the "lung volume reduction effect" in COPD patients or by the compensatory response. Accurate prediction of postoperative pulmonary function remains controversial and needs further studies.

There were several limitations for our study. First, it was a retrospective study, and therefore, the sample size was small. As a result, all of the CT scans we analyzed were ordered by respiratory physicians as a workup for COPD before inhalant treatment. Subsequently, we researched mild to moderate COPD patients only and could not compare the result with normal subjects. Our selection criteria might have

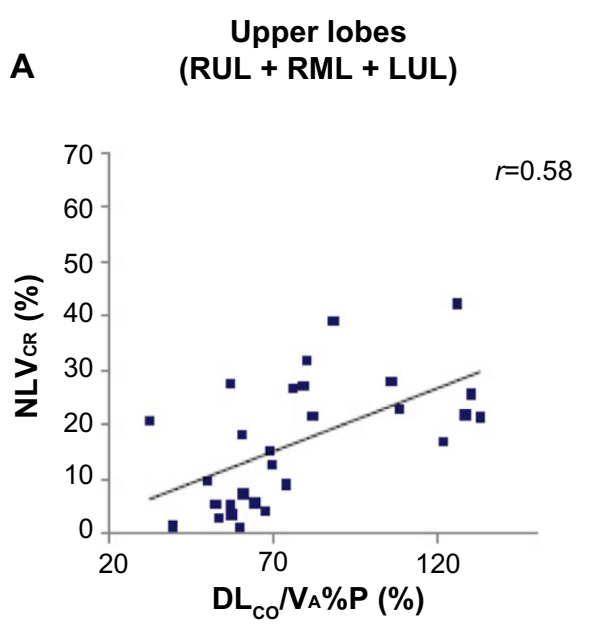

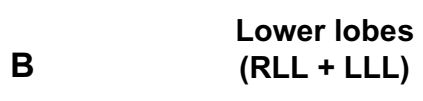

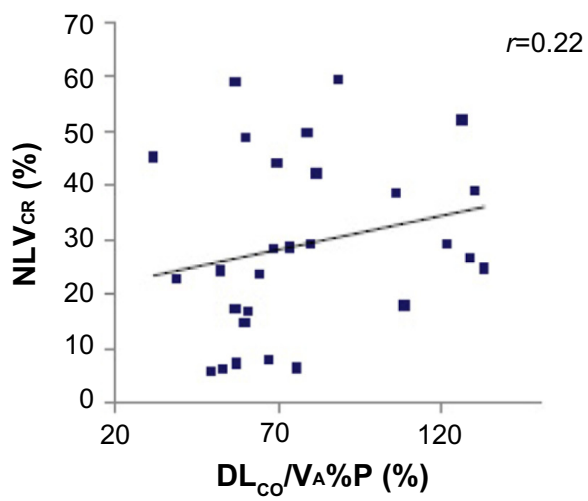

Figure 4 Correlations between $\mathrm{NLV}_{\mathrm{CR}}$ of upper lobes $(\mathbf{A})$, lower lobes $(\mathbf{B})$ and $\mathrm{DL}_{\mathrm{CO}} / \mathrm{V}_{\mathrm{A}} \% \mathrm{P}$ results.

Notes: $\mathrm{DL}_{C O} / \mathrm{V}_{\mathrm{A}} \% \mathrm{P}$ results were significantly correlated with $\mathrm{NLV} \mathrm{C}_{\mathrm{CR}}$ values for the upper lobes $(\mathbf{A})$, whereas this correlation was not significant for the lower lobes (B). Abbreviations: $\mathrm{DL}_{\mathrm{CO}} / \mathrm{V}_{\mathrm{A}} \% \mathrm{P}$, ratio of the diffusion capacity for carbon monoxide to alveolar gas volume, measured as percent predicted; LLL, left lower lobe; LUL, left upper lobe; $\mathrm{NLV}_{\mathrm{CR}}$, normal lobar volume collapsibility ratio; RLL, right lower lobe; RML, right middle lobe; RUL, right upper lobe. 


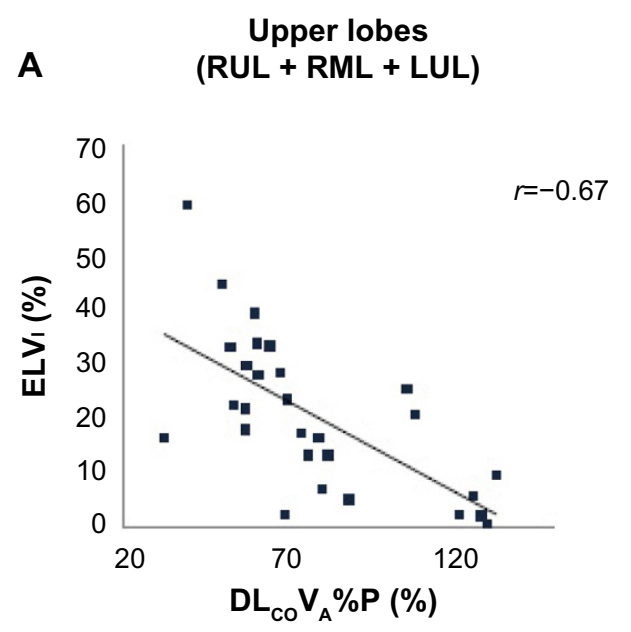

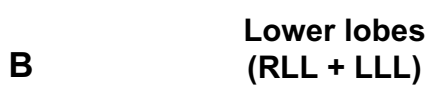

\begin{abstract}
(RLL + LLL)
\end{abstract}

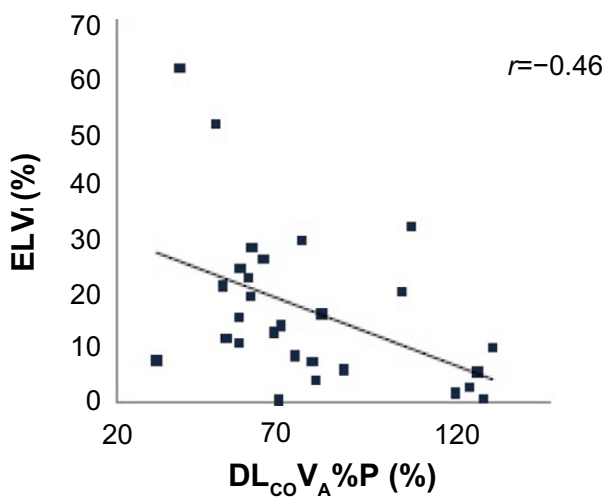

Figure 5 Correlations between ELV of upper lobes $(\mathbf{A})$ and lower lobes $(\mathbf{B})$ and $D L_{C o} / V_{A} \% \mathrm{P}$ results.

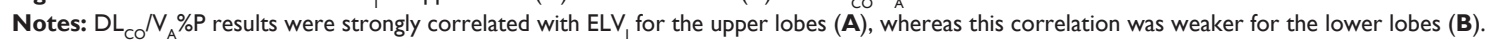

Abbreviations: $\mathrm{DL}_{\mathrm{co}} / \mathrm{V}_{\mathrm{A}} \% \mathrm{P}$, ratio of the diffusion capacity for carbon monoxide to alveolar gas volume, measured as percent predicted; ELV, inspiratory emphysematous lobar volume; LLL, left lower lobe; LUL, left upper lobe; RLL, right lower lobe; RML, right middle lobe; RUL, right upper lobe.

introduced selection bias since, as previously noted, most of our subjects in this study had mild to moderate COPD. Further investigations will be needed to determine the relationships between PFT results and NLV collapsibility indices for severe to very severe COPD patients and for normal subjects. Second, standard-dose CT protocol was used in this study. A previous report showed low-dose CT could potentially substitute for standard-dose CT on assessments in lobar volume and emphysematous volume. ${ }^{37}$ Now, we use the low-dose CT protocol, for which the exposure dose is about half that of the standarddose protocol. Third, we chose $-950 \mathrm{HU}$ as the threshold for emphysematous areas for expiratory CT. Wang et al reported that densitometry of $<-930 \mathrm{HU}$ during expiratory CT was similar to densitometry of $<-950 \mathrm{HU}$ during inspiratory $\mathrm{CT} .{ }^{38}$ We might have underestimated emphysematous volumes during expiratory CT and NLV collapsibility. However, the ideal threshold for expiratory CT is controversial, and some authors have used $-950 \mathrm{HU}$ for expiratory scan, as we did. ${ }^{12,39}$ So this is a problem that demands further studies. Fourth, our subjects were in a sitting position during PFT examinations, whereas the subjects were in a supine position during CT image acquisition. Petersson et al reported that gravity redistributed regional ventilation in the upright posture, while the influence was much less in the supine and prone posture..$^{40}$ Compared with that in a sitting position, the ventilation becomes more uniform to the craniocaudal axis, in a supine position. Thus, the collapsibility indices estimated in a spine posture might have been overestimated in the upper lobes, while they might have been underestimated in the lower lobes. Fifth, we could not evaluate wall thickness of the bronchi, which represents airway inflammation. COPD consists of emphysema and obstructive bronchitis, and bronchial wall thickness is significantly correlated with airway obstruction. ${ }^{41}$ To predict postoperative complications more accurately, additional analysis of the bronchial wall is needed.

\section{Conclusion}

Pulmonary function seemed to be different between the upper and lower lobes in COPD patients. The $\mathrm{FEV}_{1} \% \mathrm{P}$ results were correlated with NLV collapsibility indices for lower lobes, while the $\mathrm{DL}_{\mathrm{CO}} / \mathrm{V}_{\mathrm{A}} \% \mathrm{P}$ results were correlated with NLV collapsibility indices and ELV for upper lobes. Thus, evaluating lobar NLV collapsibility might be useful for estimating pulmonary function in COPD patients.

\section{Disclosure}

The authors report no conflicts of interest in this work.

\section{References}

1. Global Initiative for Chronic Obstructive Lung Disease. Global Strategy for the Diagnosis, Management, and Prevention of Chronic Obstructive Lung Disease. Vancouver, WA: Global Initiative for Chronic Obstructive Lung Disease; 2013. Available from: http://www.goldcopd.org/uploads/ users/files/GOLD_Report_2013_Feb.20.pdf. Accessed November 10, 2013.

2. Goddard PR, Nicholson EM, Laszlo G, Watt I. Computed tomography in pulmonary emphysema. Clin Radiol. 1982;33(4):379-387.

3. Bergin C, Müller N, Nichols DM, et al. The diagnosis of emphysema. A computed tomographic-pathologic correlation. Am Rev Respir Dis. 1986;133(4):541-546.

4. Bergin CJ, Müller NL, Miller RR. CT in the qualitative assessment of emphysema. J Thorac Imaging. 1986;1(2):94-103.

5. Sanders C, Nath PH, Bailey WC. Detection of emphysema with computed tomography. Correlation with pulmonary function tests and chest radiography. Invest Radiol. 1988;23(4):262-266.

6. Gevenois PA, Yernault JC. Can computed tomography quantify pulmonary emphysema? Eur Respir J. 1995;8(5):843-848. 
7. Kauczor HU, Heussel CP, Fischer B, Klamm R, Mildenberger P, Thelen M. Assessment of lung volumes using helical CT at inspiration and expiration: comparison with pulmonary function tests. AJR Am J Roentgenol. 1998;171(4):1091-1095.

8. Mergo PJ, Williams WF, Gonzalez-Rothi R, et al. Three-dimensional volumetric assessment of abnormally low attenuation of the lung from routine helical CT: inspiratory and expiratory quantification. AJR Am J Roentgenol. 1998;170(5):1355-1360.

9. Park KJ, Bergin CJ, Clausen JL. Quantitation of emphysema with three-dimensional CT densitometry: comparison with two-dimensional analysis, visual emphysema scores, and pulmonary function test results. Radiology. 1999;211(2):541-547.

10. Newell JD. Quantitative computed tomography of lung parenchyma in chronic obstructive pulmonary disease: an overview. Proc Am Thorac Soc. 2008;5(9):915-918.

11. Iwano S, Okada T, Satake H, Naganawa S. 3D-CT volumetry of the lung using multidetector row CT: comparison with pulmonary function tests. Acad Radiol. 2009;16(3):250-256.

12. Yamashiro T, Matsuoka S, Bartholmai BJ, et al. Collapsibility of lung volume by paired inspiratory and expiratory CT scans: correlations with lung function and mean lung density. Acad Radiol. 2010;17(4): 489-495.

13. Matsuo K, Iwano S, Okada T, Koike W, Naganawa S. 3D-CT lung volumetry using multidetector row computed tomography: pulmonary function of each anatomic lobe. J Thorac Imaging. 2012;27(3): 164-170.

14. Mohamed Hoesein FA, van Rikxoort E, van Ginneken B, et al. Computed tomography-quantified emphysema distribution is associated with lung function decline. Eur Respir J. 2012;40(4):844-850.

15. Come CE, Diaz AA, Curran-Everett D, et al; COPD Gene Investigators. Characterizing functional lung heterogeneity in COPD using reference equations for CT scan-measured lobar volumes. Chest. 2013;143(6): 1607-1617.

16. Schroeder JD, McKenzie AS, Zach JA, et al. Relationships between airflow obstruction and quantitative CT measurements of emphysema, air trapping, and airways in subjects with and without chronic obstructive pulmonary disease. AJR Am J Roentgenol. 2013;201(3): W460-W470.

17. Kundu S, Gu S, Leader JK, et al. Assessment of lung volume collapsibility in chronic obstructive lung disease patients using CT. Eur Radiol. 2013;23(6):1564-1572.

18. Iwano S, Kitano M, Matsuo K, et al. Pulmonary lobar volumetry using novel volumetric computer-aided diagnosis and computed tomography. Interact Cardiovasc Thorac Surg. 2013;17(1):59-65.

19. West JB. Regional differences in the lung. Chest. 1978;74(4):426-437.

20. Hogg JC. Pathophysiology of airflow limitation in chronic obstructive pulmonary disease. Lancet. 2004;364(9435):709-721.

21. Engel LA, Grassino A, Anthonisen NR. Demonstration of airway closure in man. J Appl Physiol. 1975;38(6):1117-1125.

22. Saitoh T, Koba H, Shijubo N, Tanaka H, Sugaya F. Lobar distribution of emphysema in computed tomographic densitometric analysis. Invest Radiol. 2000;35(4):235-243.

23. Parr DG, Stoel BC, Stolk J, Stockley RA. Pattern of emphysema distribution in alpha1-antitrypsin deficiency influences lung function impairment. Am J Respir Crit Care Med. 2004;170(11):1172-1178.

24. Ariyaratnam P, Loubani M, Morice AH. Hypoxic pulmonary vasoconstriction in humans. BioMed Research International. 2013;2013:623684.
25. Sekine Y, Behnia M, Fujisawa T. Impact of COPD on pulmonary complications and on long-term survival of patients undergoing surgery for NSCLC. Lung Cancer. 2002;37(1):95-101.

26. Iizasa T, Suzuki M, Yasufuku K, et al. Preoperative pulmonary function as a prognostic factor for stage I non-small cell lung carcinoma. Ann Thorac Surg. 2004;77(6):1896-1902; discussion 1902-1903.

27. Nakajima T, Sekine Y, Yamada Y, et al. Long-term surgical outcome in patients with lung cancer and coexisting severe COPD. Thorac Cardiovasc Surg. 2009;57(6):339-342.

28. Reck M, Heigener DF, Mok T, Soria JC, Rabe KF. Management of nonsmall-cell lung cancer: recent developments. Lancet. 2013;382(9893): 709-719.

29. Win T, Groves AM, Ritchie AJ, Wells FC, Cafferty F, Laroche CM. The effect of lung resection on pulmonary function and exercise capacity in lung cancer patients. Respir Care. 2007;52(6):720-726.

30. Nakahara K, Monden Y, Ohno K, Miyoshi S, Maeda H, Kawashima Y. A method for predicting postoperative lung function and its relation to postoperative complications in patients with lung cancer. Ann Thorac Surg. 1985;39(3):260-265.

31. Zeiher BG, Gross TJ, Kern JA, Lanza LA, Peterson MW. Predicting postoperative pulmonary function in patients undergoing lung resection. Chest. 1995;108(1):68-72.

32. Kushibe K, Kawaguchi T, Kimura M, Takahama M, Tojo T, Taniguchi S. Influence of the site of lobectomy and chronic obstructive pulmonary disease on pulmonary function: a follow-up analysis. Interact Cardiovasc Thorac Surg. 2009;8(5):529-533.

33. Brunelli A, Refai M, Salati M, Xiumé F, Sabbatini A. Predicted versus observed FEV1 and DLCO after major lung resection: a prospective evaluation at different postoperative periods. Ann Thorac Surg. 2007;83(3):1134-1139.

34. Rapicetta C, Tenconi S, Voltolini L, Luzzi L, Scala V, Gotti G. Impact of lobectomy for non-small-cell lung cancer on respiratory function in octogenarian patients with mild to moderate chronic obstructive pulmonary disease. Eur J Cardiothorac Surg. 2011;39(4):555-559.

35. Ueda K, Tanaka T, Hayashi M, et al. Compensation of pulmonary function after upper lobectomy versus lower lobectomy. J Thorac Cardiovasc Surg. 2011;142(4):762-767.

36. Mizobuchi T, Chen F, Yoshino I, et al. Radiologic evaluation for volume and weight of remnant lung in living lung donors. J Thorac Cardiovasc Surg. 2013;146(5):1253-1258.

37. Koyama H, Ohno Y, Yamazaki Y, et al. Quantitative and qualitative assessments of lung destruction and pulmonary functional loss from reduced-dose thin-section CT in pulmonary emphysema patients. Acad Radiol. 2010;17(2):163-168.

38. Wang X, Duan J, Du Y, Shen N, He B, Yuan H. Trapping volumetric measurement by multidetector $\mathrm{CT}$ in chronic obstructive pulmonary disease: effect of CT threshold. Med Phys. 2013;40(8):082103.

39. Zaporozhan J, Ley S, Eberhardt R, Weinheimer O, Iliyushenko S, Herth F, Kauczor HU. Paired inspiratory/expiratory volumetric thin-slice CT scan for emphysema analysis: comparison of different quantitative evaluations and pulmonary function test. Chest. 2005;128(5):3212-3220.

40. Petersson J, Rohdin M, Sánchez-Crespo A, et al. Regional lung blood flow and ventilation in upright humans studied with quantitative SPECT. Respir Physiol Neurobiol. 2009;166(1):54-60.

41. Nakano Y, Wong JC, de Jong PA, et al. The prediction of small airway dimensions using computed tomography. Am J Respir Crit Care Med. 2005;171(2):142-146. 


\section{Publish your work in this journal}

The International Journal of COPD is an international, peer-reviewed journal of therapeutics and pharmacology focusing on concise rapid reporting of clinical studies and reviews in COPD. Special focus is given to the pathophysiological processes underlying the disease, intervention programs, patient focused education, and self management protocols.

This journal is indexed on PubMed Central, MedLine and CAS. The manuscript management system is completely online and includes a very quick and fair peer-review system, which is all easy to use. Visit http://www.dovepress.com/testimonials.php to read real quotes from published authors.

Submit your manuscript here: http://www.dovepress.com/international-journal-of-copd-journal 